\title{
DOUBLE-SLOT ANTENNAS ON EXTENDED HEMISPHERICAL AND ELLIPTICAL QUARTZ DIELECTRIC LENSES
}

\author{
Daniel F. Filipovic and Gabriel M. Rebeiz \\ NASA/Center for Space Terahertz Technology \\ Electrical Engineering and Computer Science Department \\ University of Michigan \\ Ann Arbor, Michigan 48109-2122
}

Received August 16, 1993

\begin{abstract}
In this paper, the theoretical far-field patterns and Gaussianbeam coupling efficiencies are investigated for a double-slot antenna placed on quartz hemispherical lenses with varying extension lengths. The radiation patterns of the double-slot antenna are computed using ray-tracing inside the lens and electric and magnetic field integration on the spherical dielectric surface. The theoretical results are equally valid for double-dipole, log-periodic, and spiral antennas, and are presented in extension length/radius and radius/ $\lambda$. Therefore, the results yield universal design curves for quartz lenses of different diameters and at different frequencies and using different antennas. The results indicate that for single units, there exists a wide range of extension lengths (ext. length/radius $=0.61$ to 0.76 ) which result in high Gaussian-coupling efficiencies to moderately high $f / \#$ systems. For imaging array applications with high packing densities, an extension length/radius $=0.82$ to 0.93 (depending on frequency) will result in peak directivity and highest packing density but lower Gaussian-coupling efficiencies.
\end{abstract}




\section{INTRODUCTION}

This paper represents an extension of the work performed on extended hemispherical silicon lenses that was published by the authors in the October ' 93 issue of the IEEE Transactions on Microwave Theory \& Techniques [1]. In this companion paper, the computed results for an extended hemispherical lens fabricated from fused quartz are presented. The reader is referred to [1] for a complete discussion of the ray-optics/aperture-integration theory used in this analysis.

Quartz lenses can be hemispherical, hyperhemispherical, or elliptical, and many researchers have placed various antennas on these lenses for receiver applications $[2,3,4,5,6,7]$. The hyperhemispherical lens is a hemispherical lens with an attached extension length of $R / n$, where $\mathrm{n}$ is the index of refraction of the lens, and $\mathrm{R}$ is the radius of the lens [8]. The need for a full characterization of the extended hemisphere (Fig. 1) prompted us to develop a ray-optics/field-integration formulation to solve for the radiation patterns and Gaussian-coupling efficiencies of a double-slot antenna on hemispherical lenses with a varying extension length [1].

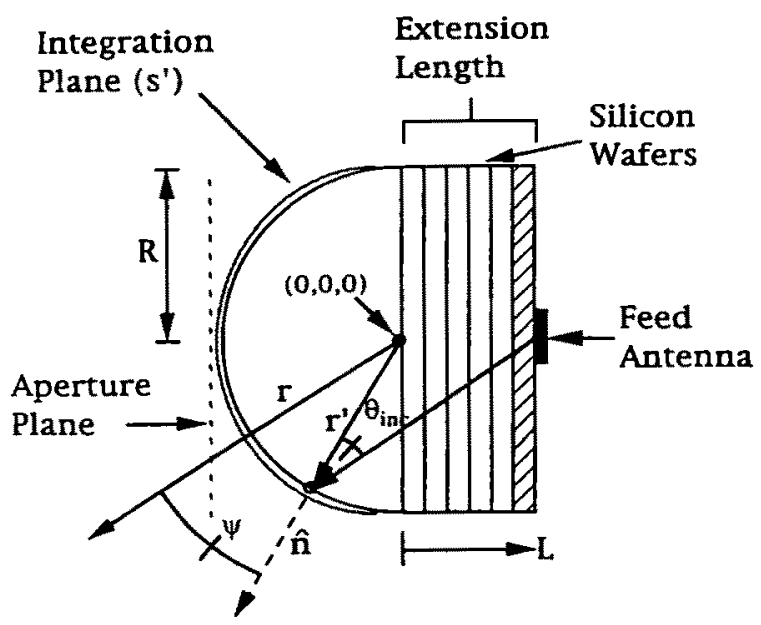

Figure 1: The extended hemispherical lens and the ray-tracing/fieldintegration technique. 
The extended hemispherical system is very practical, since it results in an antenna/lens system which couples to a wide range of quasi-optical systems simply by varying the extension length behind the hemispherical position. It is important to note that the hyperhemispherical lens is a special case of the extended hemispherical lens. Section II describes the double-slot antenna design. Section III presents the theoretical directivities and maximum "Gaussicities" for quartz extended hemispherical lenses. The term "Gaussicity" is defined as the coupling efficiency of a far-field pattern of an antenna to the far-field pattern of a Gaussian-beam. The Gaussian-coupling efficiency is defined as the total coupling efficiency which is the Gaussicity multiplied by any system losses (reflection loss, backside loss, impedance mismatch loss, etc.). Section IV shows the reflection loss with and without a matching-cap layer. Section $V$ describes the directivities and Gaussicities versus extension length as a function of frequency. The theoretical results are equally valid for double-dipole, log-periodic and spiral antennas. The only constraint is that the planar feed antenna produces a circularly symmetrical beam into the dielectric lens.

As was shown in [1], an elliptical lens can be synthesized using a hemispherical lens and attached planar wafers. For the case of a quartz lens with $\epsilon_{\mathrm{T}}=3.8$ and a diameter of $13.7 \mathrm{~mm}$, the synthesized elliptical position is $6380 \mu \mathrm{m}(\mathrm{L} / \mathrm{R}=0.93)$ behind the hemispherical point, and the hyperhemispherical position is $3520 \mu \mathrm{m}(\mathrm{R} / \mathrm{n})$ behind the hemispherical point. Recently, Büttgenbach experimentally placed a spiral antenna at a specific position behind the hyperhemispherical position (but before the elliptical position) and achieved good patterns and high aperture efficiencies [9]. Büttgenbach based his analysis on the quality of the measured far-field power patterns and did not present a full characterization of the Gaussian-coupling efficiency of an extended hemispherical lens.

\section{DOUBLE-SLOT ANTENNA DESIGN}

A double-slot antenna is chosen as the feed antenna for the quartz extended hemispherical lens. This antenna has been used previously by Kerr et al. in a $100 \mathrm{GHz}$ receiver [10] and recently Zmuidzinas built a $492 \mathrm{GHz}$ SIS receiver using a double-slot antenna on a quartz 
hyperhemispherical lens [6]. The double-slot antenna patterns are calculated assuming a sinusoidal magnetic current distribution on the slot and using an array factor in the E-plane direction. The magnetic current distribution in the slot is given by $\sin \left[k_{m}(1-|z|)\right]$ where $k_{m}$ is the mean propagation constant [11]. Figure 2 shows the calculated radiation patterns of a double-slot antenna with length $2 l=0.49 \lambda_{\text {air }}$ $\left(0.76 \lambda_{m}\right)$ and spacing $d=0.28 \lambda_{\text {air }}\left(0.43 \lambda_{m}\right)$ into a dielectric with a relative dielectric constant of $\epsilon_{r}=3.8$ (fused quartz). This design is very similar to the one used by $\mathrm{Zmuidzinas}$ in [6].

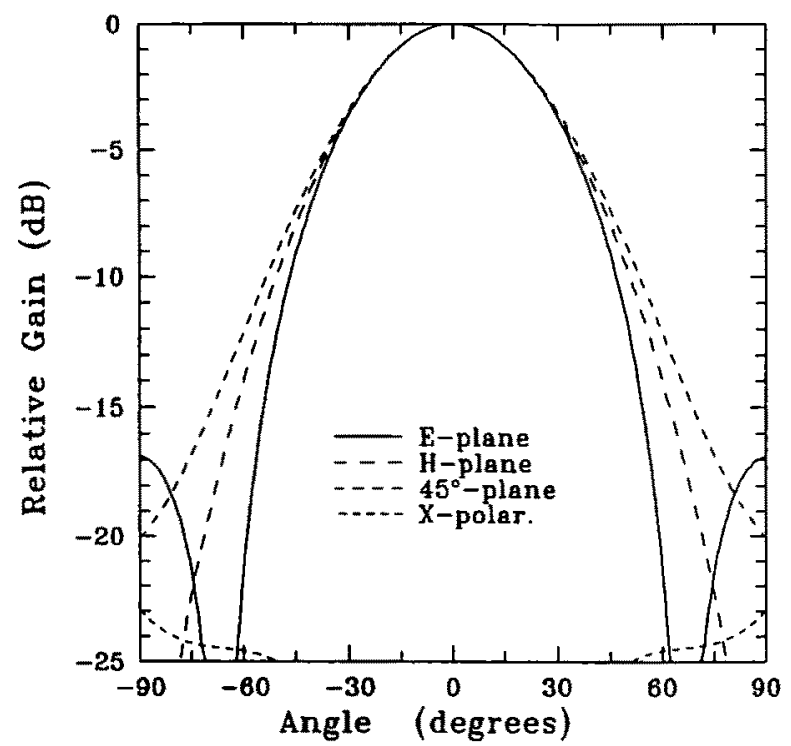

Figure 2: The radiation patterns of the double-slot antenna into a quartz $\left(\epsilon_{r}=3.8\right)$ dielectric.

The double-slot antenna results in symmetrical patterns in the infinite dielectric half space with a corresponding directivity of $11.2 \mathrm{~dB}$ and a cross-polarization level lower than $-23 \mathrm{~dB}$ in the $45^{\circ}$-plane (the directivity quoted is calculated using the pattern radiated in the dielectric only). The phase is constant across the mainlobes in the $\mathrm{E}$, $\mathrm{H}$, and $45^{\circ}$-plane, and the power radiated in this approximate cone illuminates the whole spherical surface of the extended hemispherical lenses considered in this paper. The patterns radiated to the air-side 
are broader with a $-10 \mathrm{~dB}$ beamwidth of $66^{\circ}$ in the $\mathrm{H}$-plane and a nearly uniform E-plane, and contain $28.3 \%$ of the total radiated power from the double-slot antenna. This is considerable compared to the case of a double-slot antenna on a silicon lens ( $9 \%$ power loss to the airside). The log-periodic, spiral, double-dipole antennas do not suffer from this loss due to the use of a ground-plane reflector behind the antenna. For a full treatment of log-periodic antennas including their co-polarization and cross-polarization properties, the reader is referred to [12].

\section{THEORETICAL RESULTS}

The radiation patterns from the extended hemispherical lenses are computed using a ray-tracing technique (see [1] for more detail). The double-slot antenna patterns into the dielectric are used with a ray-optics approach to calculate the distribution of the electric and magnetic fields across the spherical surface of an extended hemispherical lens (Fig. 1). The equivalent electric and magnetic current densities are then defined on the spherical surface of the lens, and the equivalent currents are integrated to yield the far-field patterns of the extended hemispherical lens.

The $\mathrm{E}$ and H-plane power patterns and the phases of the electric field in the E-plane of a $13.7 \mathrm{~mm}$ diameter lens fed by a double-slot antenna at $246 \mathrm{GHz}$ are shown for extension lengths of $3000 \mu \mathrm{m}, 3800 \mu \mathrm{m}$, $4200 \mu \mathrm{m}, 4800 \mu \mathrm{m}, 5400 \mu \mathrm{m}, 6000 \mu \mathrm{m}, 6600 \mu \mathrm{m}$, and $7200 \mu \mathrm{m}$ (Fig. 3). It is seen that the patterns become progressively narrower resulting in higher directivity up to $6000 \mu \mathrm{m}$ and then the mainlobe widens and the sidelobe levels begin to increase. It is important to note that the phase of the field is not constant in the mainlobe except around the synthesized elliptical position $(6380 \mu \mathrm{m})$. This is seen on the $6000 \mu \mathrm{m}$ plot where the phase is nearly constant in the mainlobe and then shifts $180^{\circ}$ in the first sidelobe. The corresponding peak directivity and the Gaussicity (pattern coupling efficiency) as a function of the extension length is shown in Figure 4. The Gaussicity is computed using an overlap integral between the far-field patterns and the far-field Gaussian-beam expression [1]. The directivity has a broad peak of $30.3 \mathrm{~dB}$ centered at $6000 \mu \mathrm{m}(\mathrm{L} / \mathrm{R}=0.87)$ and remains within $0.5 \mathrm{~dB}$ of the peak between $5500 \mu \mathrm{m}(\mathrm{L} / \mathrm{R}=0.80)$ and $6900 \mu \mathrm{m}(\mathrm{L} / \mathrm{R}=1.01)$. The corresponding 

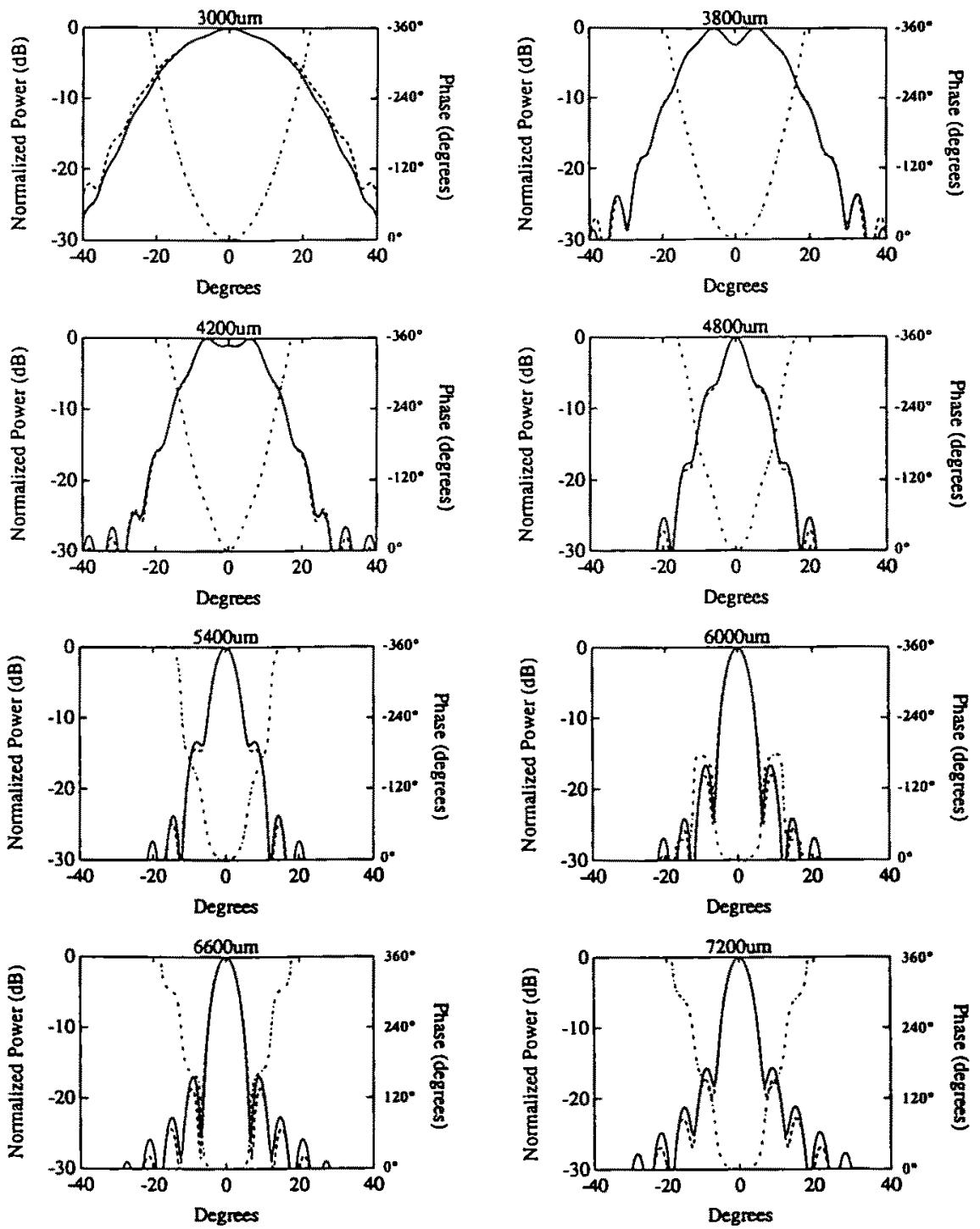

Figure 3: $E$ and H-Plane power patterns and phase in the E-plane at $246 \mathrm{GHz}$ for extension lengths of $3000 \mu \mathrm{m}, 3800 \mu \mathrm{m}, 4200 \mu \mathrm{m}, 4800 \mu \mathrm{m}$, $5400 \mu \mathrm{m}, 6000 \mu \mathrm{m}, 6600 \mu \mathrm{m}$, and $7200 \mu \mathrm{m}$. The $(-\cdot-\cdot-)$ line corresponds to the phase. 
aperture efficiency (coupling to a plane wave) peaks at $86 \%$. This peak position agrees well with Büttgenbach's formulation on the diffractionlimit of a lens (see [9] for more detail). In the far-field, the decrease in Gaussicity at the larger extension lengths can be interpreted as the result of the formation of out-of-phase sidelobes whose level progressively increases as the extension length is increased. It is seen that the Gaussicity is above $92 \%$, up to $4600 \mu \mathrm{m}(\mathrm{L} / \mathrm{R}=0.67)$ and drops to $84-82 \%$ at the peak directivity and synthesized elliptical positions.

\section{$L / R$}

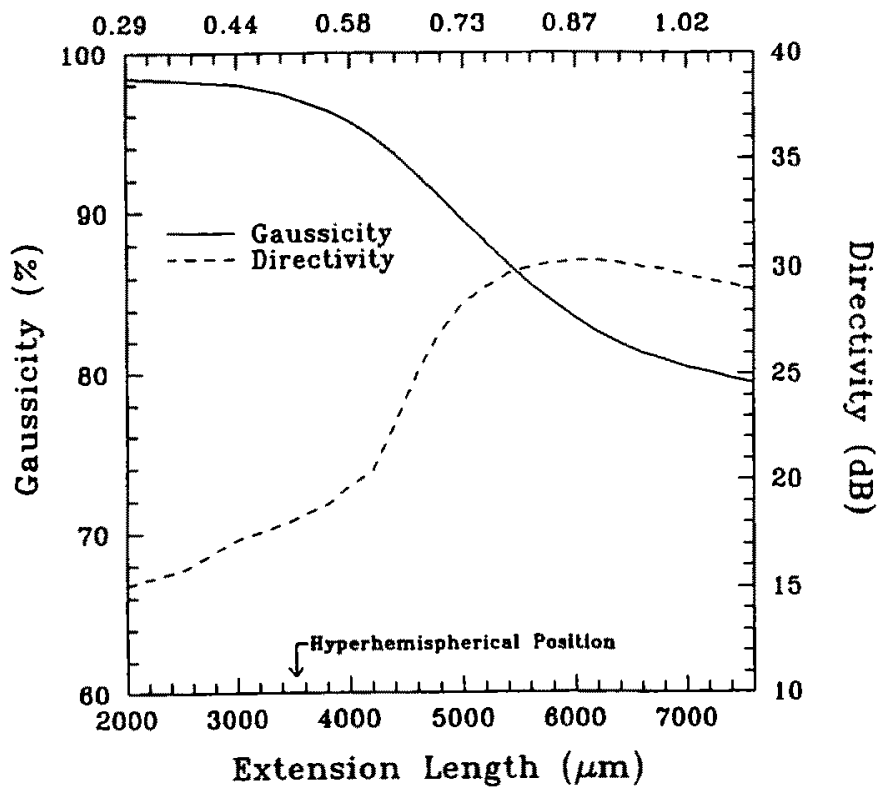

Figure 4: Directivity and maximum Gaussicity (pattern coupling efficiency) as a function of extension length at $246 \mathrm{GHz}$.

A similar analysis of a $13.7 \mathrm{~mm}$ diameter lens was performed at $100 \mathrm{GHz}$ and $500 \mathrm{GHz}$, assuming the same radiation patterns of the feed antenna. Figure 5 shows the patterns and phases for extension lengths of $3000 \mu \mathrm{m}, 3800 \mu \mathrm{m}, 4200 \mu \mathrm{m}, 4800 \mu \mathrm{m}, 5400 \mu \mathrm{m}, 6000 \mu \mathrm{m}, 6600 \mu \mathrm{m}$, and $7200 \mu \mathrm{m}$ at $500 \mathrm{GHz}$. The peak directivity (not the directivity at boresight) and Gaussicity calculations at $100 \mathrm{GHz}, 246 \mathrm{GHz}$ and $500 \mathrm{GHz}$ are shown in Figure 6. It is important to note that the $500 \mathrm{GHz}$ patterns 

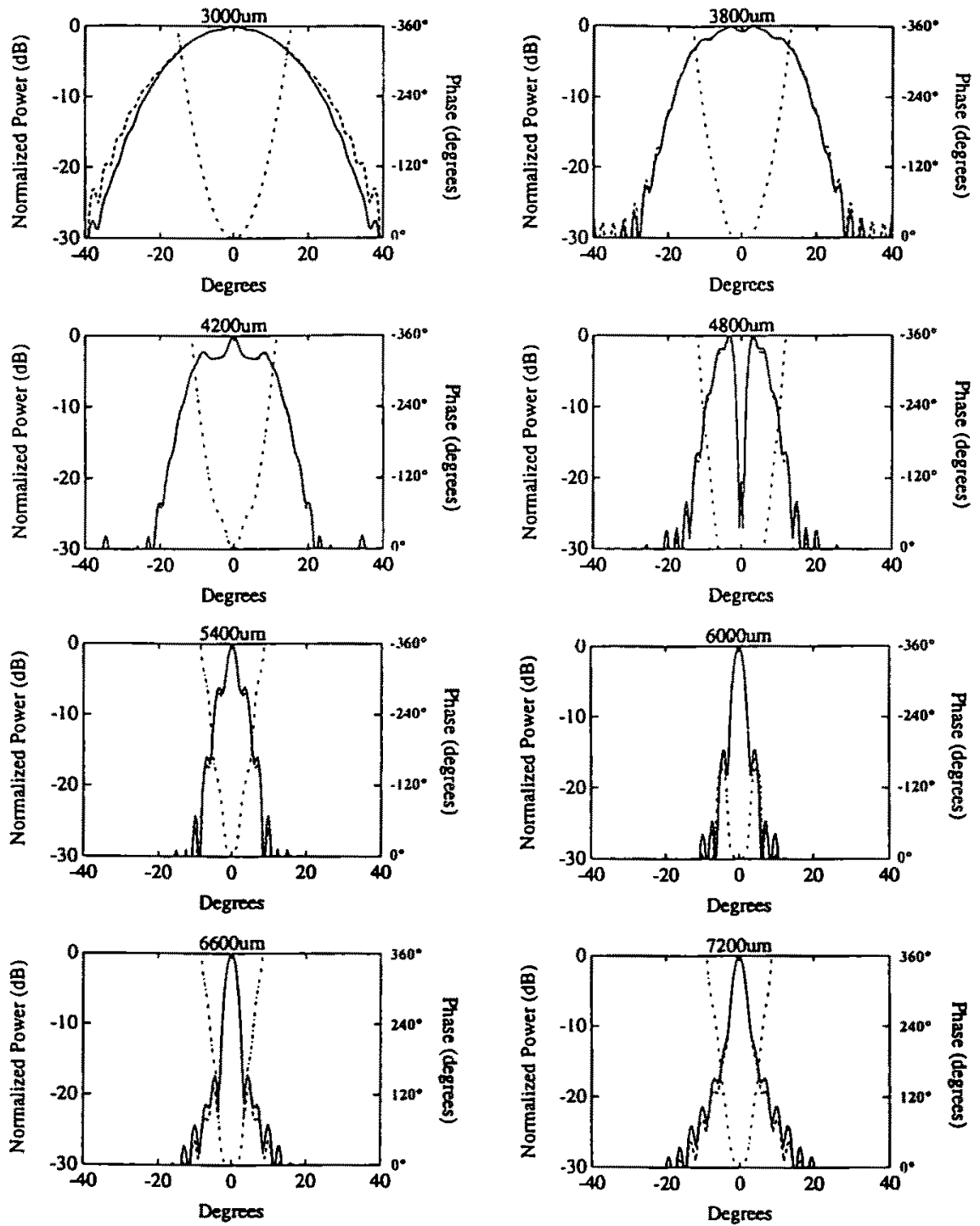

Figure 5: $E$ and H-Plane power patterns and phase in the E-plane at $500 \mathrm{GHz}$ for extension lengths of $3000 \mu \mathrm{m}, 3800 \mu \mathrm{m}, 4200 \mu \mathrm{m}, 4800 \mu \mathrm{m}$, $5400 \mu \mathrm{m}, 6000 \mu \mathrm{m}, 6600 \mu \mathrm{m}$, and $7200 \mu \mathrm{m}$. The (-.-.-) line corresponds to the phase. 


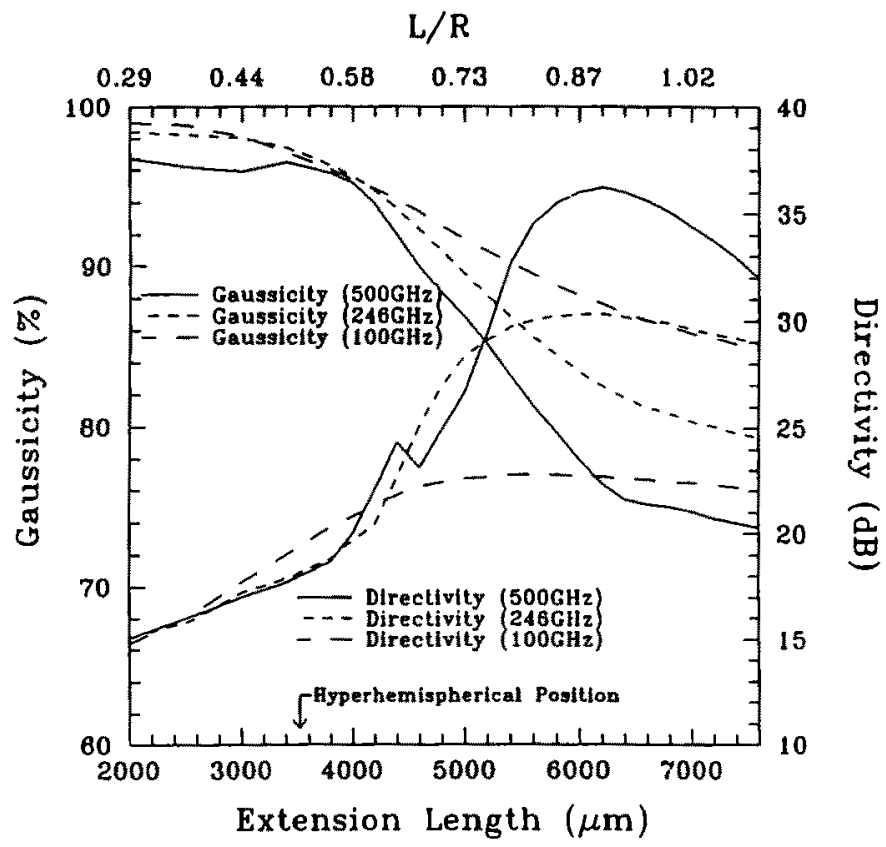

Figure 6: Directivity and maximum Gaussicity (pattern coupling efficiency) as a function of extension length at $100 \mathrm{GHz}, 246 \mathrm{GHz}$, and $500 \mathrm{GHz}$.

at positions of $4200 \mu \mathrm{m}(\mathrm{L} / \mathrm{R}=0.61)$ and $4800 \mu \mathrm{m}(\mathrm{L} / \mathrm{R}=0.70)$ do yield a high Gaussicity. This is counter-intuitive, especially at $4800 \mu \mathrm{m}$ where a dip of $-20 \mathrm{~dB}$ exists at $\theta=0$. The reason for the high Gaussicity is that the phase is quadratic and that there is very little power in a pattern around $\theta=0$. The quadratic phase matches well to a converging Gaussian-beam. Patterns with dips and peaks around $\theta=0$ have been judged before by researchers to be "quite bad". This example shows that both the amplitude and the phase of the far-field pattern are important to completely judge a pattern and its coupling efficiency to Gaussian-beams. At $100 \mathrm{GHz}$, the peak in the directivity curve occurs at $5600 \mu \mathrm{m}(\mathrm{L} / \mathrm{R}=0.82)$ and is very broad. The corresponding Gaussicity is very smooth with a drop of $9 \%$ at $5600 \mu \mathrm{m}$ and $12 \%$ at the synthesized elliptical position. On the other hand, at $500 \mathrm{GHz}$, the directivity curve shows a distinct peak of $36.2 \mathrm{~dB}$ centered at $6200 \mu \mathrm{m}(\mathrm{L} / \mathrm{R}=0.90)$, with a corresponding aperture efficiency of $81 \%$. Again, this position agrees 
well with Büttgenbach's diffraction method (see [9] for more detail). Note that at higher frequencies, the peak directivity is closer to the synthesized elliptical position, which is the result of the geometrical optics approximation becoming more accurate. The Gaussicity peaks at $500 \mathrm{GHz}$ at the hyperhemispherical position $(3520 \mu \mathrm{m})$ with a value of $97 \%$ and drops to $76 \%$ at the $6200 \mu \mathrm{m}-6400 \mu \mathrm{m}$ position. Again, as the frequency increases, the ray-optics approximation becomes more valid, and this explains the peak in Gaussicity at the hyperhemispherical position.

The near-field waist and radius of curvature are found from an inverse Fourier transform of the far-field Gaussian-beam [1] (Fig. 7). These are referenced to the aperture at the tip of the lens (Fig. 1). As expected, the radius of curvature is infinity at the synthesized elliptical position $(6380 \mu \mathrm{m})$ and changes sign immediately afterwards. This

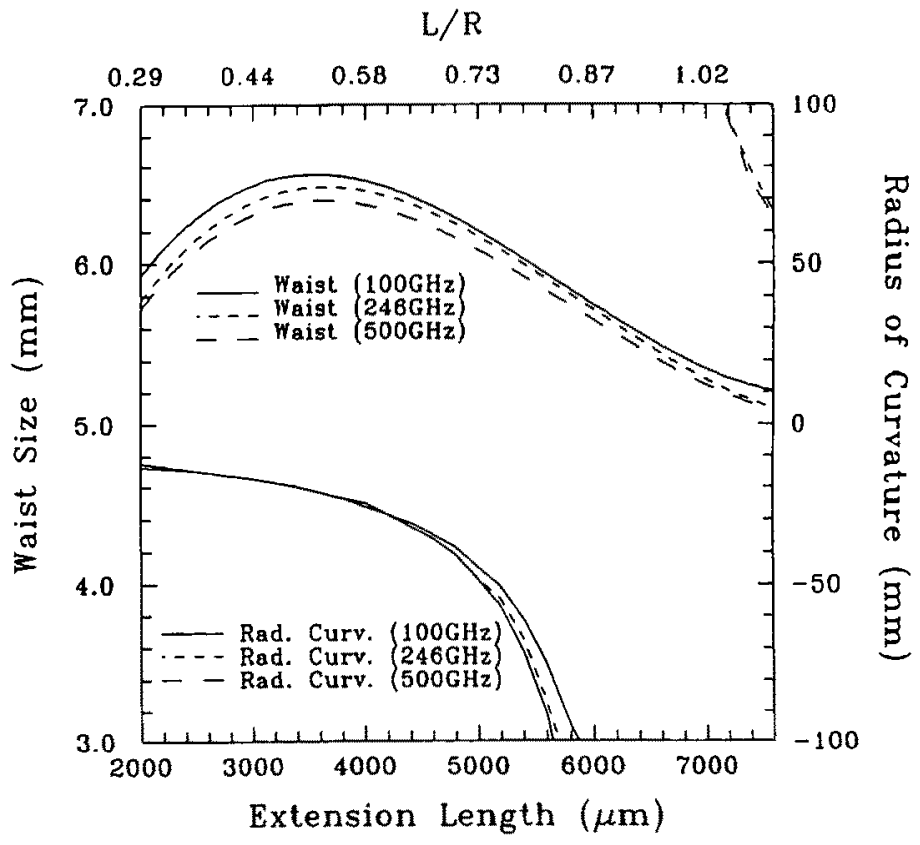

Figure 7: Waist and radius of curvature as a function of extension length at $100 \mathrm{GHz}, 246 \mathrm{GHz}$, and $500 \mathrm{GHz}$. 
means that the synthesized elliptical lens is a good approximation to a true elliptical lens and couples best to a Gaussian-beam at its minimum waist. As expected, the Gaussian-beam radius of curvature approaches the lens radius $(6.858 \mathrm{~mm})$ when the extension length is small. The beam waist is nearly constant at $6.1 \pm 0.4 \mathrm{~mm}$ for extension lengths between $2000 \mu \mathrm{m}(\mathrm{L} / \mathrm{R}=0.29)$ and $6000 \mu \mathrm{m}(\mathrm{L} / \mathrm{R}=0.87)$ for $100 \mathrm{GHz}$, $246 \mathrm{GHz}$, and $500 \mathrm{GHz}$. It is mainly the radius of curvature that is changing from $-12 \mathrm{~mm}$ to $-\infty$ between $2000 \mu \mathrm{m}$ and $6380 \mu \mathrm{m}$ for all three frequencies. Therefore, the converging Gaussian-beam must be very well characterized on the lens aperture for optimal Gaussian-coupling efficiency.

The directivity and Gaussicity are calculated for a true elliptical lens using the same approach as for the extended hemispherical lens at $246 \mathrm{GHz}$. The true elliptical lens is defined such that the minor axis (perpendicular to boresight) is equal to the radius of the extended hemispherical lens, so that the effective area on the aperture plane is the same for both lenses (see [1] for more detail). The E and H-plane mainlobes are slightly narrower than those of the synthesized elliptical lens and have $0.5 \mathrm{~dB}$ higher sidelobes. The directivity is $30.7 \mathrm{~dB}$ which is $0.5 \mathrm{~dB}$ higher than the synthesized ellipse. The elliptical lens Gaussicity is $83 \%$ and is independent of frequency. The corresponding minimum waist at the aperture plane is $5.7 \mathrm{~mm}$, which is $0.2 \mathrm{~mm}$ more than that of the synthesized elliptical position.

\section{REFLECTION LOSS CALCULATIONS}

The ray-tracing technique presented earlier also results in a full characterization of the reflection loss at the lens-air interface as a function of the extension length (Fig. 8). The reflection loss is calculated by finding the total power radiated into the quartz dielectric by the double-slot antenna and dividing it by the transmitted power which is integrated over the outside spherical surface of the lens. At low extension lengths or for high directivity feed antennas, the calculated loss is $0.5 \mathrm{~dB}$ which is the same as that predicted from simple transmission-line theory, $\left(\frac{n-1}{n+1}\right)^{2}$, where $n$ is the index of refraction of the fused quartz lens. For a double-slot antenna, with a directivity of $11.2 \mathrm{~dB}$, the loss increases to $2.7-3.2 \mathrm{~dB}$ at the peak-position and synthesized elliptical position due to the unfocused power lost in the rectangular extension 


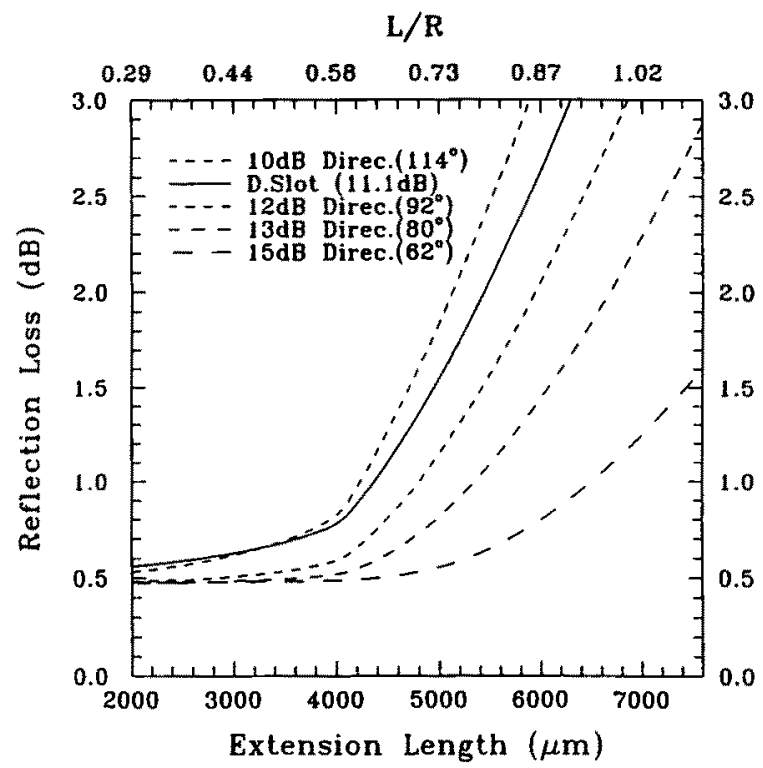

(a)

$\mathrm{L} / \mathrm{R}$

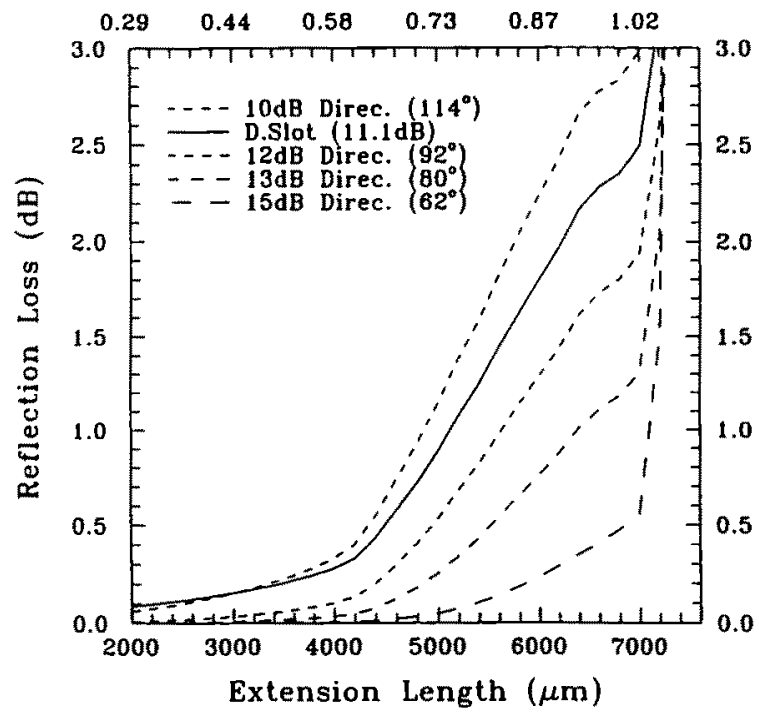

(b)

Figure 8: Reflection loss at the lens/air interface as a function of the extension length for different feed antenna directivities (or corresponding 10dB Gaussian-pattern beamwidths): (a) without a matching layer and (b) with a $\lambda_{m} / 4$ matching cap layer. 
section and from the total internal reflection of wide angle rays (above $57^{\circ}$ ). This is a considerable amount of reflected power which will appear in the sidelobe structure and the back-side power pattern of the extended hemispherical lens antenna. This power is assumed in this paper as a total loss in terms of Gaussian-coupling efficiency. The power lost to the rectangular extension section is considerable for extension lengths near the synthesized elliptical position. This presents a serious limitation for using low index of refraction lenses which require long extension lengths. On the other hand, a quartz elliptical lens has a reflection loss of only $0.65 \mathrm{~dB}$, since the whole surface of the lens is properly curved.

The Gaussian-coupling efficiency of the extended hemispherical system must be multiplied by the reflection loss to result in a complete characterization of the system. Note that for feed antennas with greater than 13dB directivity (or a 10dB Gaussian-pattern beamwidth of less than $80^{\circ}$ into the quartz lens), the reflection loss does not increase as much since most of the power is near normal incidence to the lens. The directivities of spiral and log-periodic antennas on a quartz dielectric and backed by a ground-plane reflector are not known but are expected to be between $12 \mathrm{~dB}$ and $13 \mathrm{~dB}$. The directivity of a double-dipole antenna inside the dielectric (as presented by Skalare [7]) is also around $12 \mathrm{~dB}$. Therefore, these three antennas present a much lower reflection loss at the quartz-air interface. This is very important at high frequencies (above $300 \mathrm{GHz}$ ) where it is difficult to manufacture an accurate matching layer for the quartz lens. For a quartz elliptical lens, the reflection losses decrease to less than $0.5 \mathrm{~dB}$ for antenna directivities greater than $13 \mathrm{~dB}$.

Figure 8 also includes the reflection loss using a uniformly thick $\lambda_{m} / 4$ matching cap layer with dielectric constant equal to $\sqrt{\epsilon_{\text {lens }}}$. The reflection loss for the double-slot antenna (directivity $=11.2 \mathrm{~dB}$ ) is $0.2 \mathrm{~dB}$ at the hyperhemispherical position, and increases to $2.2 \mathrm{~dB}$ at the synthesized elliptical position. Again, this is primarily due to power lost in the rectangular extension section. The radiation patterns and the directivities remain essentially the same with the matching cap layer. As expected, the reflection loss for the higher directivity antennas (12$15 \mathrm{~dB}$ ) are lower (Fig. 8). No attempt is made here to design an optimum matching layer by varying the thickness of the matching dielectric 
across the surface of the lens. It is expected that an elliptical lens with a $\lambda_{m} / 4$ matching layer will result in a reflection loss of less than $0.2 \mathrm{~dB}$ for all antenna directivities (11-15dB).

\section{GAUSSICITY VS. FREQUENCY}

A comparison of the directivity and Gaussicity for the true elliptical lens, the synthesized elliptical lens, and an extended hemispherical lens at peak directivity position for $100 \mathrm{GHz}-1 \mathrm{THz}$ is shown in Fig. 9. The true elliptical lens has a constant Gaussicity of $83 \%$ and a quadratic increase in the directivity, as expected from ray-optics calculations. The directivity of the synthesized elliptical lens shows a $1.7 \mathrm{~dB}$ drop at $1 \mathrm{THz}$ compared to the elliptical lens due to the residual phase error on the planar aperture. Similarly, the Gaussicity drops to $70 \%$ at $1 \mathrm{THz}$ due

Aperture Radius $\left(R / \lambda_{\text {air }}\right)$

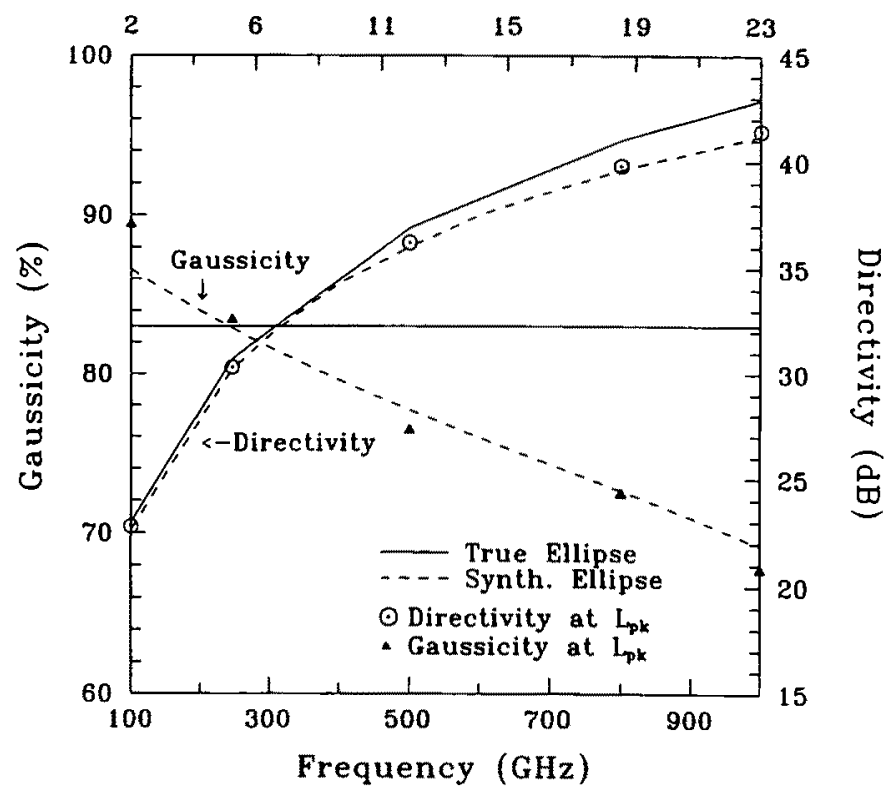

Figure 9: Directivity and maximum Gaussicity (pattern coupling efficiency) as a function of frequency (or lens radius) for a true elliptical lens, a synthesized elliptical lens, and an extended hemispherical lens at peak directivity position $\left(\mathrm{L}_{\mathrm{pk}}\right)$. 
to the same effect. The directivities at extension lengths resulting in peak directivity $\left(\mathrm{L}_{\mathrm{pk}}\right)$ for $100 \mathrm{GHz}, 246 \mathrm{GHz}, 500 \mathrm{GHz}, 800 \mathrm{GHz}$, and $1 \mathrm{THz}$ are also presented in Figure 9. Figure 10 shows that $\mathrm{L}_{\mathrm{pk}}$ asymptotically approaches the synthesized elliptical position with increasing radius/wavelength (or increasing frequency for a constant radius). The directivity at $\mathrm{L}_{\mathrm{pk}}$ is always slightly higher $(0.1-0.2 \mathrm{~dB})$ than the synthesized elliptical directivity and shows a $1.5 \mathrm{~dB}$ drop at $1 \mathrm{THz}$ from the true elliptical lens. The Gaussicities at $L_{p k}$ are the same as those of the synthesized elliptical position, except at $100 \mathrm{GHz}$, where the peak directivity position is closer to the hyperhemispherical position.

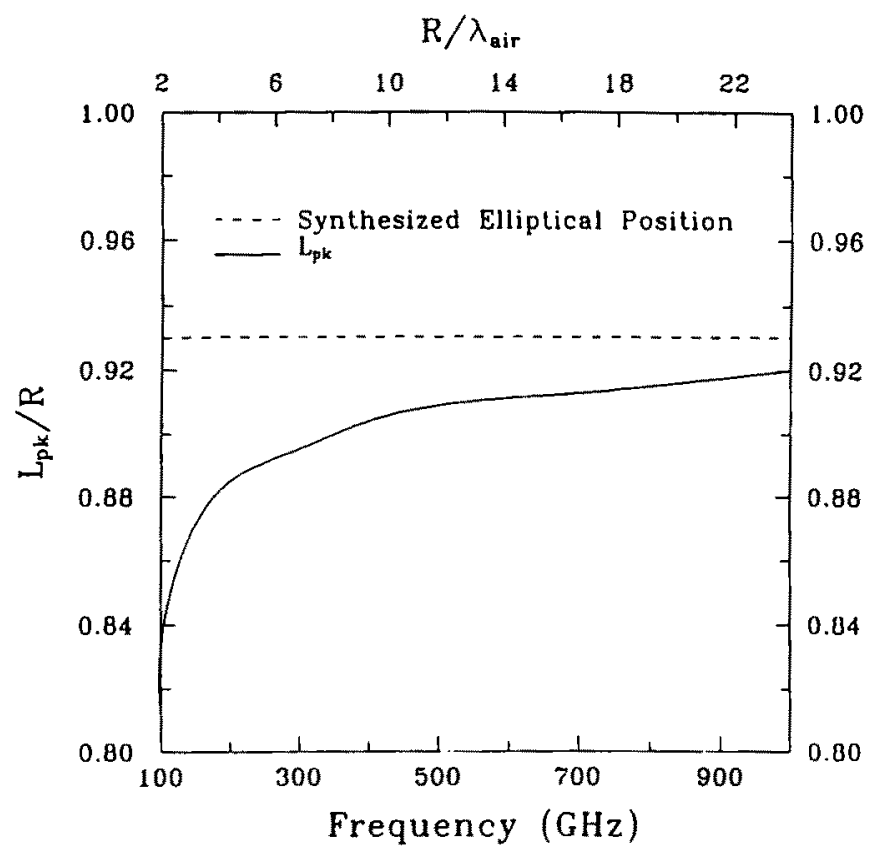

Figure 10: Extension length for peak directivity versus frequency (or $R / \lambda)$.

The Gaussicity and directivity for extension lengths of $3520 \mu \mathrm{m}$ (hyperhemispherical), $5000 \mu \mathrm{m}$ (an intermediate position), and $6380 \mu \mathrm{m}$ (synthesized ellipse) are shown in Figure 11. The hyperhemispherical lens results in a nearly constant directivity of $18 \mathrm{~dB}$ and Gaussicity of $97 \%$. Notice that the directivity is constant with frequency due 
to the constant $\mathrm{n}^{2}$ magnification of the hyperhemispherical lens. The Gaussicity at $5000 \mu \mathrm{m}$ is slightly lower than the hyperhemispherical lens and the directivity increases to around $30 \mathrm{~dB}$ at high frequencies. The Gaussicity at $6380 \mu \mathrm{m}$ (close to the synthesized elliptical position) is the lowest of all three positions, but results in the largest directivity. The Gaussicity at the synthesized elliptical position (and at $\mathrm{L}_{\mathrm{pk}}$ ) drops to $70 \%$ at $1 \mathrm{THz}$ while the Gaussicity for the $5000 \mu \mathrm{m}$ position drops to $80 \%$ at the same frequency. The hyperhemispherical position shows the highest theoretical Gaussicity. However, it is hard to experimentally achieve this efficiency at the hyperhemispherical position due to the difficulty in aligning to the strongly converging beams needed at the aperture of the lens [1]. Thus the region between $4200 \mu \mathrm{m}$ and $5200 \mu \mathrm{m}$ $(\mathrm{L} / \mathrm{R}=0.61$ to 0.76$)$ results in the best compromise between alignment, directivity and Gaussian-coupling efficiency.

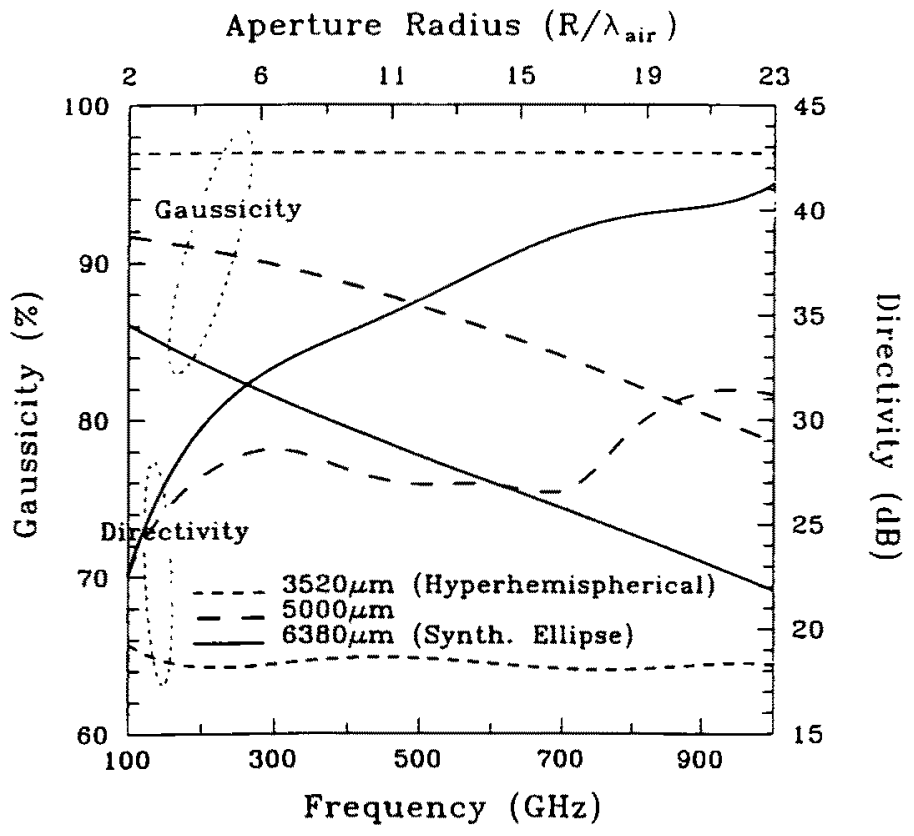

Figure 11: Directivity and Gaussicity (pattern coupling efficiency) as a function of frequency (or lens radius) for an extended hemispherical lens at $L=3520 \mu \mathrm{m}, 5000 \mu \mathrm{m}$, and $6380 \mu \mathrm{m}$. 


\section{DISCUSSION AND CONCLUSION}

In this paper, a ray-optics/field-integration approach has been used to fully characterize an extended hemispherical lens system with a double-slot antenna feed. The results show that the directivity is strongly dependent on the extension length, especially at high frequencies. Our calculations also show that a $13.7 \mathrm{~mm}$ diameter quartz lens at the synthesized elliptical position can be used up to about $1 \mathrm{THz}$ (or $\mathrm{R} / \lambda=23$ ) with an associated directivity greater than $40 \mathrm{~dB}$, but with a drop in the Gaussicity to $70 \%$.

The question arises of where to place the antenna on a lens for the best possible performance. The answer, according to our calculations, depends on the specific application (for example, a single unit or a unit in an imaging array). For single units, the hyperhemispherical position is not the best place since it results in a low directivity and requires small $f / \#$ systems that are difficult to align. In our opinion, one should use an extension length that results in an acceptably high directivity and therefore a large $f / \#$ for the objective lens. This also yields an easy-to-align quasi-optical system. However, the high directivity is achieved at the expense of a reduction in the Gaussiancoupling efficiency. For a $13.7 \mathrm{~mm}$ diameter quartz lens, we believe that a good compromise between the increase in directivity and the decrease in Gaussian-coupling efficiency is achieved at $4200 \mu \mathrm{m}-5200 \mu \mathrm{m}$ extension length ( $\mathrm{L} / \mathrm{R}=0.61$ to 0.76 ). The corresponding Gaussicity is very high being always above $80 \%$ for frequencies below $1 \mathrm{THz}$. Also, the corresponding dielectric reflection loss with a matching-cap layer is acceptable (0.3-1.0dB). The Gaussian-coupling efficiency for a double-slot antenna at a position between $4200 \mu \mathrm{m}$ to $5200 \mu \mathrm{m}(\mathrm{L} / \mathrm{R}=0.61$ to 0.76$)$ is $60-50 \%$, which includes the reflection loss at the quartz-air interface with a $\lambda_{m} / 4$ matching layer (0.4-1.2dB) and the back-side power loss $(28 \%)$. The Gaussian-coupling efficiency for double-dipole and spiral antennas is expected to be higher than double-slot antennas due to the absence of power loss to the air-side and lower reflection loss at the lens-air interface (see Fig. 8).

For imaging arrays, the extension length resulting in peak directivity should be chosen so as to result in the best possible packing density in the focal plane. For a $13.7 \mathrm{~mm}$ diameter lens, the aper- 
ture efficiency (coupling to a plane wave) at $\mathrm{L}_{\mathrm{pk}}$ is $84 \%$ at $246 \mathrm{GHz}$ $(\mathrm{R} / \lambda=5.6), 77 \%$ at $500 \mathrm{GHz}(\mathrm{R} / \lambda=11.4)$, and $63 \%$ at $1 \mathrm{THz}(\mathrm{R} / \lambda=23)$. The aperture efficiency vs. frequency (or $R / \lambda$ ) can be calculated using the formula $\epsilon_{\mathrm{ap}}=\left(\frac{\lambda^{2}}{4 \pi}\right)\left(\frac{\mathrm{D}}{\mathrm{A}}\right)$, where $\mathrm{D}$ is the directivity (Fig. 9) and A is the aperture area of the lens. The corresponding Gaussicity is high at $246 \mathrm{GHz}$ with a value of $84 \%$ and drops to $70 \%$ at $1 \mathrm{THz}$. However at $\mathrm{L}_{\mathrm{pk}}$, the reflection loss at the lens-air interface is high, about $3 \mathrm{~dB}$ without a matching-cap layer, and $2.4 \mathrm{~dB}$ with a $\lambda_{m} / 4$ matching-cap layer. This reflection loss is mainly due to power lost in the flat extension section, and it is possible to reduce the reflection loss with a higher directivity feed antenna (spiral antenna, log-periodic antenna, or double-dipole antenna all with a backing ground plane). The Gaussiancoupling efficiency of a double-slot antenna on a quartz lens with a $\lambda_{m} / 4$ matching-cap layer at the $\mathrm{L}_{\mathrm{pk}}$ position is therefore $30 \%$. This is considerably lower than the $70 \%$ Gaussian-coupling efficiency expected for double-slot antennas on extended hemispherical silicon lenses [1] at the $\mathrm{L}_{\text {pk }}$ position with a matching-cap layer. In [9], Büttgenbach found a high Gaussian-coupling efficiency to a telescope (no absolute efficiency data were quoted) with the use of an exended hemispherical quartz lens at $L_{p k}$ position. The reason is that he used a spiral antenna and a back-plane reflector, thereby eliminating the back-side power loss (28\%) and considerably reducing the reflection loss due to the increase in the directivity (12-13dB) of the planar antenna inside the lens.

As seen from our calculations, double-slot antennas on extended hemispherical quartz dielectrics suffer from two major loss factors. One is the power loss to the air side (28\%), which can be eliminated with the use of a double-dipole, spiral, or log-periodic antenna and a back reflector. The other is the high reflection loss at larger extension lengths $\left(L_{p k}\right.$ or synthesized elliptical positions). Zmuidzinas measured an estimated $60 \%$ Gaussian-coupling efficiency at $492 \mathrm{GHz}$ because he used a hyperhemispherical lens with a low extension length. His $f / \#$ was low and he used an additional plastic lens to collimate the beam. The loss in the Gaussian-coupling efficiency due to the additional lens is currently being investigated [13]. The reflection loss calculations in Fig. 8 are also applicable to double-dipole, spiral, and log-periodic antennas, and are not as high as the double-slot antenna. One way to considerably reduce the reflection losses for all these antennas is through the use of 
a true elliptical lens. The elliptical lens results in $0.6-0.65 \mathrm{~dB}$ reflection loss without a matching cap layer and a maximum of $0.2 \mathrm{~dB}$ with a $\lambda_{m} / 4$ matching cap layer. Another way is through the use of a high dielectric constant lens (silicon, alumina, etc.), which require shorter extension sections. This also eliminates the need of a back-plane reflector in the case of double-dipole, spiral, and log-periodic antennas thereby resulting in truly wideband designs.

\section{ACKNOWLEDGEMENTS}

This work was supported by the NASA/Center for Space Terahertz Technology at the University of Michigan. We are very grateful to Dr. Thomas Büttgenbach, Prof. Jonas Zmuidzinas and Prof. David Rutledge, all at the California Institute of Technology, for their immense contributions to this project.

\section{REFERENCES}

[1] D.F. Filipovic, S.S. Gearhart, and G.M. Rebeiz, "Double-slot antennas on extended hemispherical and elliptical silicon dielectric lenses," IEEE Trans. on Microwave Theory Tech., October, 1993.

[2] D.B. Rutledge, D.P. Neikirk and D.P. Kasilingam, "Integrated circuit antennas," Infrared and Millimeter-Waves, vol. 10, K.J. Button, Ed., New York: Academic Press, pp. 1-90, 1983.

[3] M.J. Wengler, D.P. Woody, R.E. Miller, and T.G. Phillips, "A low noise receiver for millimeter and submillimeter wavelengths," Int. J. Infrared and Millimeter Waves, vol. 6, pp. 697-706, 1985.

[4] Q. Hu, Z.A. Mears, P.L. Richards, and S.L. Loyd, "Millimeter-wave quasioptical SIS mixers," IEEE Trans. Magn., vol. 25, pp. 1380-1383, 1989.

[5] T.H. Büttgenbach, "A fixed tuned broadband matching structure for submillimeter SIS receivers," IEEE Trans. on Applied Superconductivity, vol. 2, no. 3, pp. 165-175, 1992.

[6] J. Zmuidzinas, "Quasi-optical slot antenna SIS mixers," IEEE Trans. on Microwave Theory Tech., vol. MTT-40, no. 9, pp. 1797-1804, 1991.

[7] A. Skalare, H. van de Stadt, Th. de Graauw, R.A. Panhuyzen, M.M.T.M. Dierichs, "Double Dipole Antenna SIS Receivers at 100 and $400 \mathrm{GHz}$," Third 
Int. Symp. on Space Terahertz Technology, Ann Arbor, MI, pp. 222-233, March 1992.

[8] M. Born and E. Wolf, Principles of Optics . New York: Permagon Press, pp. 252-252, 1959.

[9] T.H. Büttgenbach, "An improved solution for integrated array optics in quasi-optical $\mathrm{mm}$ and submm receivers: the hybrid antenna," to appear in the IEEE Trans. on Microwave Theory Tech., October, 1993.

[10] A.R. Kerr, P.H. Siegel, and R.J. Mattauch, "A simple quasi-optical mixer for 100-120GHz," IEEE-MTT Int. Microwave Symp. Digest, pp. 96-98, 1977.

[11] M. Kominami, D.M. Pozar, and D.H. Schaubert, "Dipole and slot elements and arrays on semi-infinite substrates," IEEE Trans. Antennas Propagat., vol. AP-33, pp. 600-607, June 1985.

[12] B.K. Kormanyos, C.C. Ling, and G.M. Rebeiz, "A planar wideband millimeter-wave subharmonic receiver," in IEEE/MTT-S Int. Microwave Symp. Dig., 1991, pp.213-216; also IEEE Trans. Microwave Theory Tech., to be published (Oct. 1993).

[13] Zmuidzinas, private communication, 1993. 